\title{
Digitalised, decentralised power infrastructures challenge blockchains
}

1 Geert Deconinck PhD, CEng, FIET, SMIEEE

Full Professor, EnergyVille, Department of Electrical Engineering, KU Leuven, Leuven, Belgium (Orcid:0000-0002-2225-3987)

(corresponding author: geert.deconinck@kuleuven.be)
2 Floris Vankrunkelsven MSc, CEng Researcher, EnergyVille, Department of Electrical Engineering, KU Leuven, Leuven, Belgium
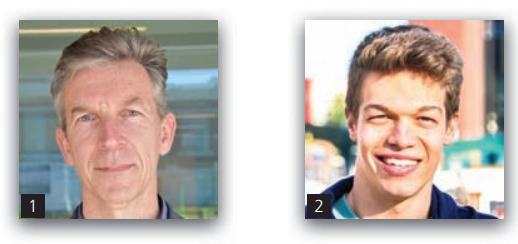

Electricity generation is decentralising quickly. Simultaneously, final energy use for residential customers electrifies in order to reduce carbon dioxide emissions. Together with ubiquitous digitalisation, this decentralisation and flexibility at the demand side paves the way towards local energy communities and - in its most distributed version - to peer-to-peer energy trading, where customers buy and sell electricity among each other. Although peer-to-peer energy trading is not yet legal everywhere, 'citizen energy communities' have been introduced as cornerstones of the energy transition by the European Commission in their 'Clean Energy for All Europeans' programme. This paper firstly discusses this digitalisation and decentralisation of the power infrastructure. These trends are supported by distributed information technologies, including peer-to-peer control paradigms. Distributed ledger technologies, such as blockchains, might be one such piece of the puzzle. The second part of the paper investigates whether blockchain technologies, and their associated smart contracts, offer advantages for larger-scale peer-to-peer energy-trading applications over a classic, centralised approach. Different blockchain implementations are investigated and qualitatively evaluated from a scalability, efficiency and trust perspective. The conclusion indicates that in the current state of the art, a trade-off between decentralised and more classical (hierarchically centralised) solutions suits larger-scale peer-to-peer energy-trading applications best.

\section{Introduction}

The electric power infrastructure and the information infrastructure become more and more interwoven. Digitalisation and decentralisation are key drivers both in the supply of energy services and in the underlying communication and control infrastructure. Such interdependent trends and technological changes are driving the co-evolution of the infrastructure systems associated with the supply of and demand for energy services to meet societal needs and expectations. As an example, evolutions in information technologies and trends towards local (decentralised) electricity generation and storage motivate local energy communities to trade excess electricity within their neighbourhood (leading to peer-to-peer energy trading). In non-energy applications, blockchain technology is often well positioned to manage and record such peer-to-peer transactions. This paper investigates whether blockchains are able to overcome the challenges related to a larger-scale peer-to-peer energy-trading application as well.

The paper is structured as follows. Section 2 elaborates the trends related to the digitalisation of the energy system, with its associated opportunities and threats. Section 3 focuses on the decentralisation of the energy system, where energy communities are highlighted as a trend, but where the legislative framework is often lagging behind. It also introduces peer-to-peer interaction as a relevant decentralised smart energy application. Section 4 introduces the blockchain technology in this application context. Section 5 tailors possible blockchain implementations for the specific case of peerto-peer energy trading, and it evaluates these choices qualitatively from a scalability, efficiency and trust perspective.

The major findings include the following.

- Larger-scale peer-to-peer energy-trading applications benefit from off-loading the proof of work from smart meter nodes such that the less scalable consensus mechanism runs only on full nodes (which could be run by the distribution system operator (DSO)).

- Because of their distributed nature and the ability to record energy-trading transactions correctly, in case that a majority of full nodes are honest, blockchain implementations are more trustful than centralised approaches. Additionally, blockchains are also immutable.

- From a privacy perspective, the transparency of transactions recorded on the blockchain conflicts with privacy requirements, such as the right to be forgotten.

The conclusion indicates that in the current state of the art, a trade-off between decentralised and more classical (hierarchically 
centralised) solutions suits larger-scale peer-to-peer energy-trading applications best.

\section{Digitalisation of the energy system}

\subsection{Trends}

The energy system digitalises. In the past decades, information and communication technologies have been inserted into many infrastructures, including energy systems. They basically allow for a better monitoring than in a purely analogue world and also for remote control; they are turning the passive electric power system into a smart grid.

A smart grid has been defined by the European Technology Platform for Electricity Networks of the Future as 'an electricity network that intelligently integrates the actions of the users connected to it, both classical generators as well as consumers, and consumers that are producing themselves - so-called prosumers -, in a way to efficiently integrate sustainable economic and secure electricity supply' (Scott, 2009: p. 14). In such a smart grid, two infrastructures operate jointly, the information and communication infrastructure for monitoring and control and the electrical infrastructure for the power grid applications.

Such digitalisation is ongoing in many economic sectors: through the digitisation of technical processes, business processes are digitalised. It is driven by the increasing amount of communication bandwidth available and the standardisation of communication technologies towards Transmission Control Protocol/Internet Protocol as a communication protocol for exchange of information. Data are generated by sensors that can be embedded everywhere, resulting in an Internet of things. Data can be processed by ever-smaller devices (miniaturisation) and can be stored locally or in the cloud.

All these trends also diffuse into the electric power infrastructure, from the digital substation in the grid backbone towards smart meters at the grid edge and beyond into the home energy systems (Liu et al., 2016). For instance, smart thermostats at home learn the residents' local comfort settings and the building characteristics in order to control the electric heat pump optimally. It allows avoiding power peaks, increasing comfort or savings on the energy bill through efficient heating control. By way of different communication technologies, one can remotely monitor appliances in real time and can implement manual control actions or automated demand response applications (Deconinck and Thoelen, 2019). Smart meters (i.e. digital meters with communication abilities) are measuring consumption data at fine granularity, connected to home energy systems as well as external applications, allowing for data-driven control. At the grid side, the DSO remotely monitors and controls the grid elements in substations or on feeders. For grid operations, one is able to forecast the electricity generation by photovoltaics and by wind energy accurately. At the market level, automated market clearing and automatic auctioning are implemented.

\subsection{Opportunities}

Such datafication and digitalisation create opportunities, particularly if open data are used, which are freely available to many different users, by way of a standardised interface, and under pre-agreed conditions. Innovations occur if open data from different sources get integrated. Figure 1 shows an example from electricityMap (2020),
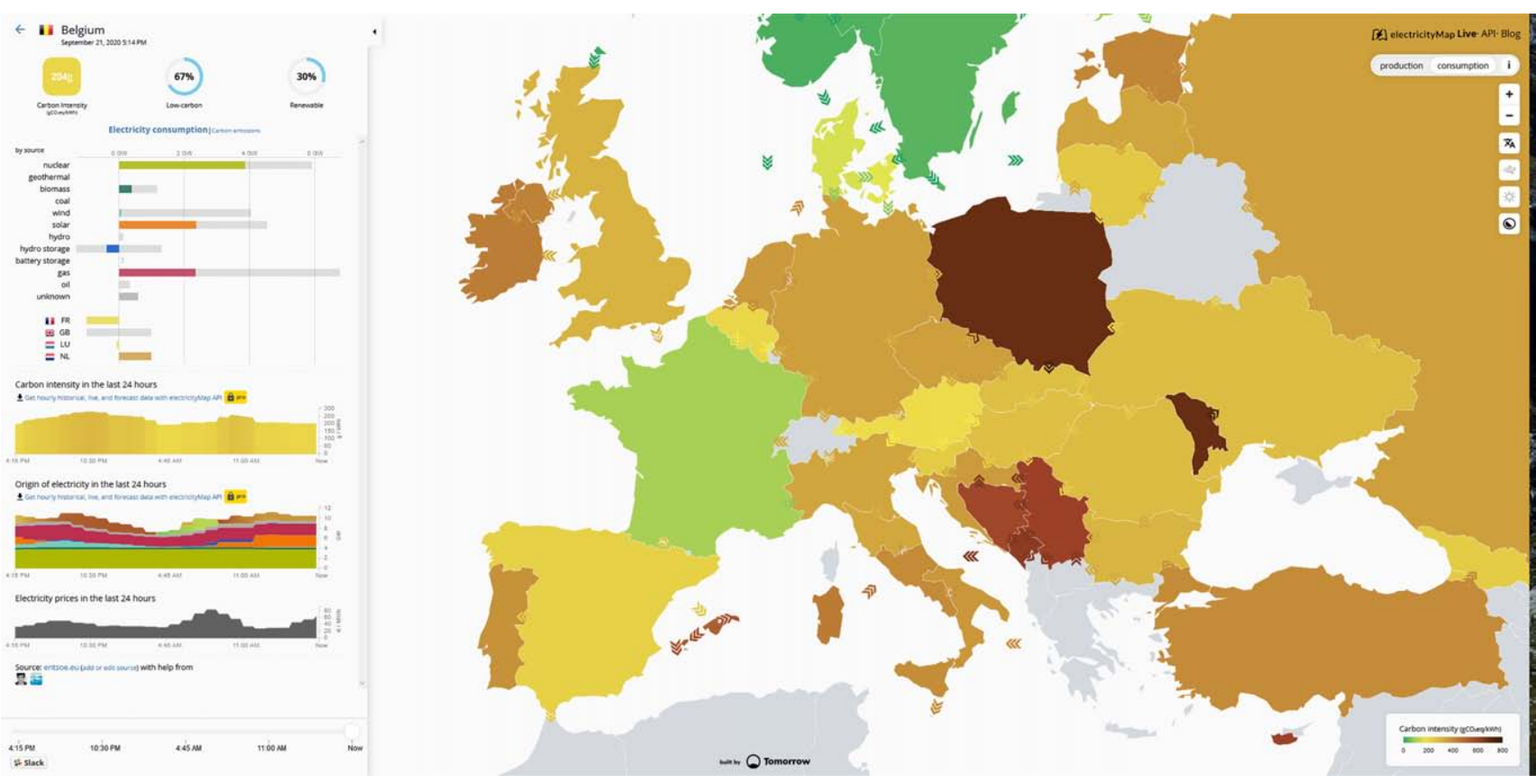

Figure 1. Carbon intensity of electricity consumption (electricityMap, 2020) 
an app and website that collects data about the carbon dioxide $\left(\mathrm{CO}_{2}\right)$ intensity ('carbon intensity') of electricity production or consumption. Power system data from all over the world are processed and visualised. The dynamically updated information corresponds to the amount of carbon dioxide equivalent emissions (in $t / k W h$ ) for the electricity consumed in a country. The colour coding is from green, climate-friendly countries to yellowish or brownish, high-fossilcontent electricity production or consumption.

Hence, thanks to such open data, community projects can collect data from different sources; big data approaches can turn them into usable information for informing policymakers and interested citizens.

\subsection{Threats}

However, there is also a downside to these data applications, as they might reveal personal or privacy-sensitive data. For instance, Figure 2 provides a time series of the electric power usage at a particular home, captured with a high-resolution metering system. Analysing the data based on individual appliance characteristics allows identifying the time of use of a washing machine, an oven and a coffee machine. Going further into detail, one can even derive the set points of the washing machine (low or high temperature, cotton wash against delicate programme) (Labeeuw and Deconinck, 2011). According to Europe's General Data Protection Regulation (GDPR) (EC, 2016), many precautions need to be taken in order to process such sensitive data, including prior consent of the data owner.

Fully integrating privacy in, for example, smart metering applications puts severe restrictions: if the goal of metering is only to be able to provide a monthly electricity bill, there is no need to record data every $15 \mathrm{~min}$, not even daily. Analogously, if the DSO needs to make feeder load profiles, individual data are not relevant but aggregated data are enough. Privacy-invading habits are widely spread: if the consumer makes a supply contract with their electricity retailer, why would the retailer need to know the consumer's gender, age or social security number? They only need to know whether the consumer is a major who is able to sign a contract legally (e.g. 18+ years). Thus, a lot of attention is needed in order to make digitalised grid applications privacyfriendly and to take into account all data protection requirements.

Digitalisation also creates other threats - for example, endangering customer protection. If a customer is not paying an electricity bill, a supplier or DSO is technically able to disconnect this customer from the grid remotely by way of the smart meter. Customer protection legislation - for example, in Flanders, Belgium - requires that customers always remain connected to electricity even if they have unpaid bills, albeit at a low power rating $(10 \mathrm{~A}$ at $230 \mathrm{~V})$, which allows the customer to either cook something or heat up the building a bit (Flemish Government, 2013). Such regulation is needed in order to protect the customer. Additionally, customer protection legislation implies that one is able to check locally on the smart meter whether the bill from the supplier is correct. Therefore, the metrological function of a smart meter - being able to collect data on how much electricity one has been using - should be checkable by the end consumer and has priority over the remotely collected data. Often such legislative frameworks are running behind the actual technological possibilities of the smart grid.

The digitalisation of the energy system, with interconnected intelligent devices, also creates vulnerabilities. It makes the

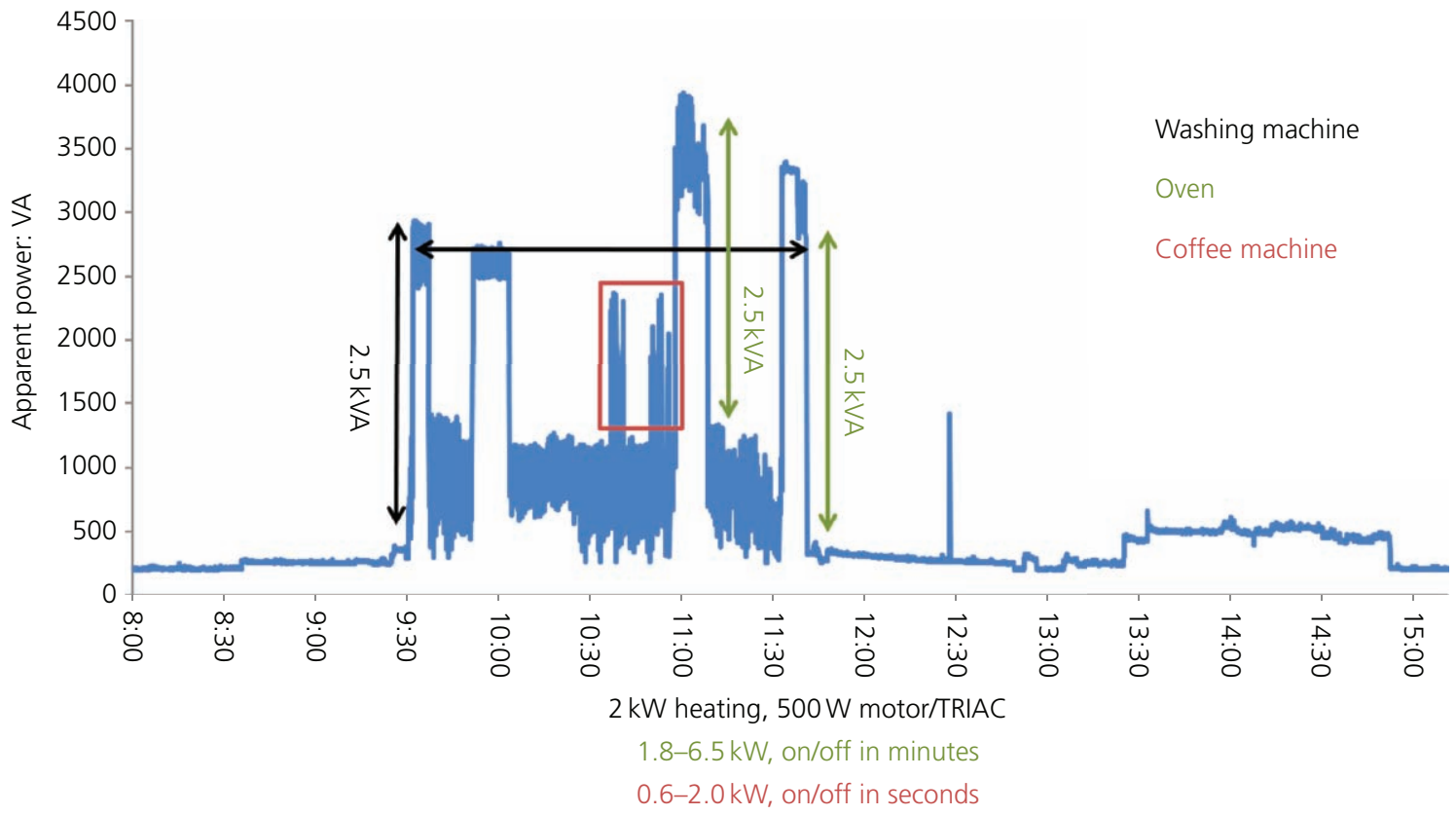

Figure 2. Appliance detection from high-resolution metering data. TRIAC, triode for alternating current (Labeeuw and Deconinck, 2011) 
infrastructure vulnerable to cyberattacks, and hence also the society, because of its dependence on electricity as an energy carrier. Attack examples include the BlackEnergy malware (Khan et al., 2016) attack that has put part of Ukraine in the dark in December 2015 or the Industroyer attack (Cherepanov and Lipovsky, 2017) in December 2016 in Ukraine as well. Accessing such hacking tools is not too difficult: websites such as SCADAhacker (2020) or the European hacker organisation Chaos Computer Club e.V. (CCC, 2020) raise awareness or even include links to relevant hacking tools.

Hence, the digitalisation of the energy system is both a blessing and a curse, and cybersecurity protection should be taken into account from the design phase of smart grid applications. For better resilience, more than one protection layer is needed. A defence in depth requires multiple levels that enforce confidentiality, integrity and availability properties. Beyond customer protection regulations and GDPR compliance, privacy-by-design approaches are needed.

\section{Decentralisation of the energy system}

Besides digitalisation, decentralisation is an important trend for the energy system, both for generation and for control.

Electricity generation decentralises by the widespread introduction of photovoltaic installations; also, heat is often produced close to where it is consumed.

\subsection{Distributed control and energy communities}

This decentralisation paves the way towards local energy communities, where a group of households or consumers organise themselves independently from a supplier or DSO or where they share some energy assets (e.g. a battery storage system or some electricity-generation devices). Such energy communities are often organised in energy co-operatives as legal entities.

Approximately 1500 of these European energy co-operatives, representing about 1000000 citizens, are gathered in the Renewable Energy Cooperatives (REScoop) (REScoop.eu, 2019a) (Figure 3). REScoop describes energy communities as 'a way to organise citizens that want to cooperate together in an energysector related activity based on open and democratic participation and governance, so that the activity can provide services or other benefits to the members or the local community. [...] The primary purpose of energy communities is to create social innovation - to engage in an economic activity with non-commercial aims' (REScoop.eu, 2019a). As an example, some of the co-operatives invest in photovoltaic or wind generation for their participants or organise joint buying of insulation material to improve energy efficiency. Others co-operate with social housing companies to provide energy services or to renovate the building stock.

\subsection{Legislative framework}

Although some forms of fully decentralised interaction (e.g. directly selling electricity to one's neighbour over the public domain) are not (yet) allowed from a regulatory perspective in many countries, it is clearly the way forward. At the EU level, this vision has been set out in 2018 by the European Commission in its 'Clean Energy for All Europeans' package, which defines 'citizen energy communities' as one of the cornerstones in the
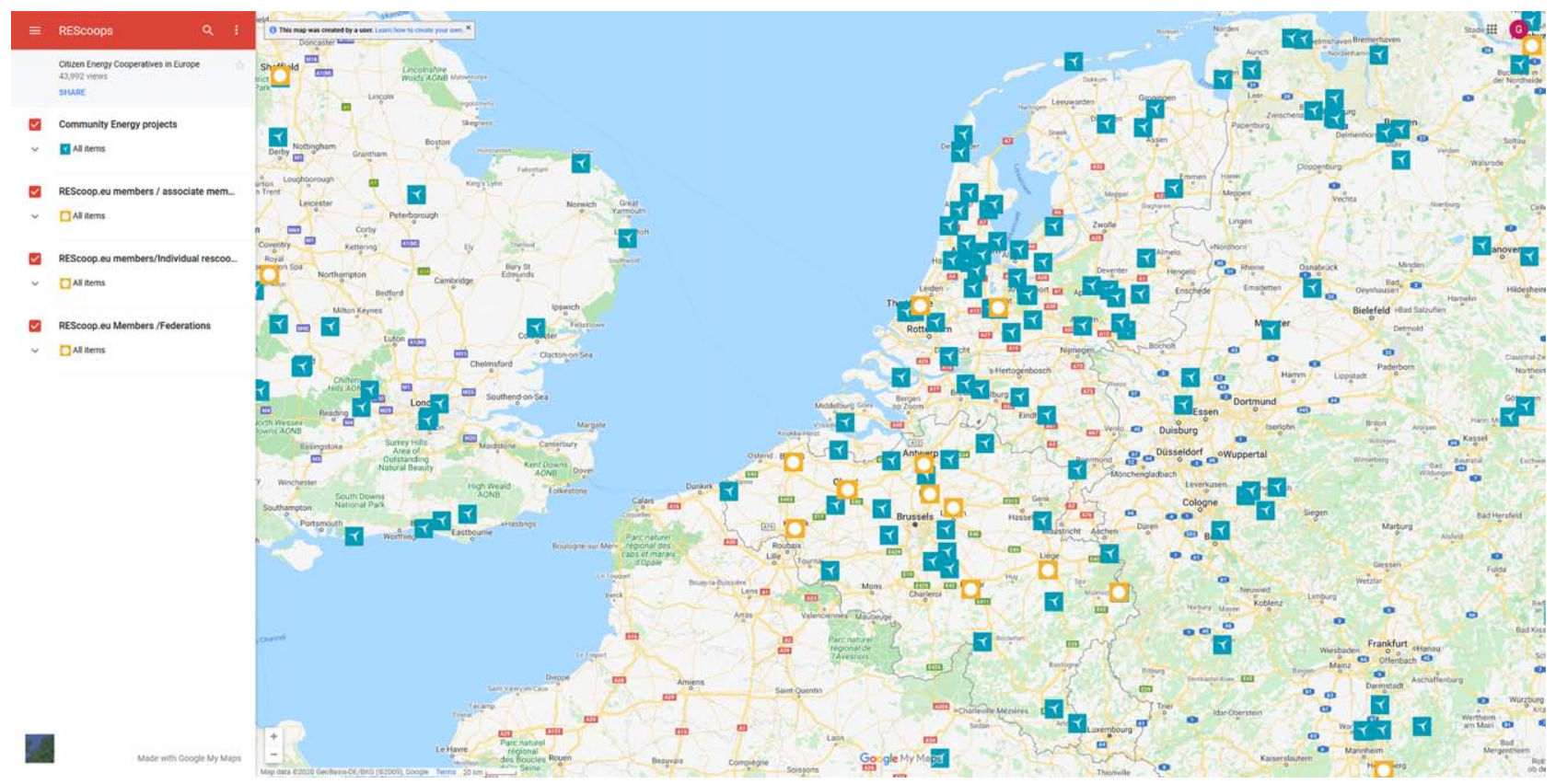

Figure 3. Diagram of energy co-operatives in the Belgium, Netherlands and Luxembourg area that are member of REScoop (REScoop.eu, 2019a) (20 GeoBasis-DE/BKG (@2009), Google) 
energy transition. Citizen energy communities are explicitly mentioned in article 2(11) of the recast electricity directive (EU/ 2019/944) (EC, 2019). They are defined as 'a legal entity that:

- is based on voluntary and open participation and is effectively controlled by members or shareholders that are natural persons, local authorities, including municipalities, or small enterprises;

- has for its primary purpose to provide environmental, economic or social community benefits to its members or shareholders or to the local areas where it operates rather than to generate financial profits; and

- may engage in generation, including from renewable sources, distribution, supply, consumption, aggregation, energy storage, energy efficiency services or charging services for electric vehicles or provide other energy services to its members or shareholders'. (EC, 2019: p. L158/140)

As a side note, in that same regulatory package, also renewable energy communities are defined in the recast renewable energy directive (EU/2018/2001) (EC, 2018), in article 2(16). See the briefing by REScoop.eu (2019b) for the subtle differences between these 'citizen energy communities' and 'renewable energy communities'.

Much of the European policy needs to be translated into countryspecific legislation, and not all European member states are equally front-running in the energy transition. Roberts and Gauthier (2019) reviewed all national energy and climate plans (NECPs) of the European member states for their view on energy communities, and they conclude, as an understatement, that there is 'much room for improvement' (Roberts and Gauthier, 2019: p. 11-12). Their key takeaways are that awareness in EU member states on energy communities is moderate but actual planning is very low. At the positive end, a few member states (e.g. Greece) show strong commitment and their NECPs have a comprehensive treatment of energy communities, including defined targets and detailed policies and measures. At the negative end, other member states (e.g. Estonia, Germany, Malta and Sweden) completely ignore energy communities in their NECPs. In between, many NECPs suffer from ambiguity around energy communities and they fail to distinguish them from distinct activities such as (individual) self-consumption. They conclude by stating that in the NECPs, 'renewable energy communities and self-consumption overshadow other dimensions where energy communities can contribute: energy efficiency, energy poverty, ownership of distribution network, e-mobility, rural development, district heating, etc.' (Roberts and Gauthier, 2019: p. 2).

Often, energy co-operatives or local energy communities are actively looking for a role in this emerging energy landscape. Often DSOs as regulated entities - take up a rather conservative role, and these innovative communities have to find their place between existing players (e.g. suppliers or retailers) and newcomers (energy service providers, aggregators, flexibility service providers etc.). The evolution towards local energy communities also pushes control towards lower and more decentralised levels.
This trend towards decentralisation and distributed control is not only a European trend. Many rural areas are electrified using microgrids. Examples include the Project for Renewable Energy in Rural Markets initiatives in Argentina (Argentina.gob.ar, 2019) or the Mee Panyar project in Myanmar (community-based minigrid operation) (Mee Panyar, 2019) and many other projects in remote areas or in developing countries. This way, it helps contribute to UN Sustainable Development Goal 7, 'affordable and clean energy' (UNDP, 2015).

\subsection{Peer-to-peer interaction}

In the most decentralised form of distributed control of electric power systems, peer-to-peer approaches are found, where each unit is autonomous and collaborates directly with its neighbours, without a central actor. More than a decade ago, it has been shown - both in simulations and in a practical implementation - that such completely distributed approaches are possible by controlling a microgrid with distributed energy resources as an autonomous electricity network without any central master (Vanthournout et al., 2005). In that microgrid, control was implemented as a combination of local droop control at the inverters, with a decentralised secondary control (to keep the voltage within its limits), and a decentralised tertiary control application (that prioritises the more economic energy resources). The communication infrastructure was based on a peer-to-peer gossiping protocol that ran on top of a dynamic semantic overlay network (Deconinck and Vanthournout, 2009). Later work shows that peer-to-peer control, communication and trading can be efficiently combined (Almasalma et al., 2019; Haapola et al., 2018).

A survey of peer-to-peer energy-trading approaches was recently compiled by Abdella and Shuiab (2018). In a peer-to-peer energytrading system, customers buy and sell energy directly among each other, without suppliers intervening, as shown in Figure 4. It has several advantages: as electricity is balanced locally and on a realtime basis, fewer grid assets are used, reducing losses. For final customers, it is assumed to be cheaper to buy electricity from their neighbour than from their supplier. Similarly, when selling excess energy, they achieve a higher return from their neighbours than when selling it back to the supplier or grid operator (Esat, 2019).

In order to evaluate better the value of peer-to-peer energy trading and of energy communities, the Users Technology Cooperation Programme of the International Energy Agency launched in 2019 'Global Observatory on Peer-to-peer, Community Selfconsumption and Transactive Energy Models' as an international forum 'to understand the policy, regulatory, social and technological conditions necessary to support the wider deployment of peer-to-peer, community self-consumption and transactive energy models' (UsersTCP, 2020).

Finally, with these energy communities also come new value chains and actors (Montakhabi et al., 2020), as well as technological challenges. The rest of this paper focuses on such technological challenges, as elaborated by Deconinck et al. (2019) and Vankrunkelsven (2019). The paper will specifically focus on peer-to- 


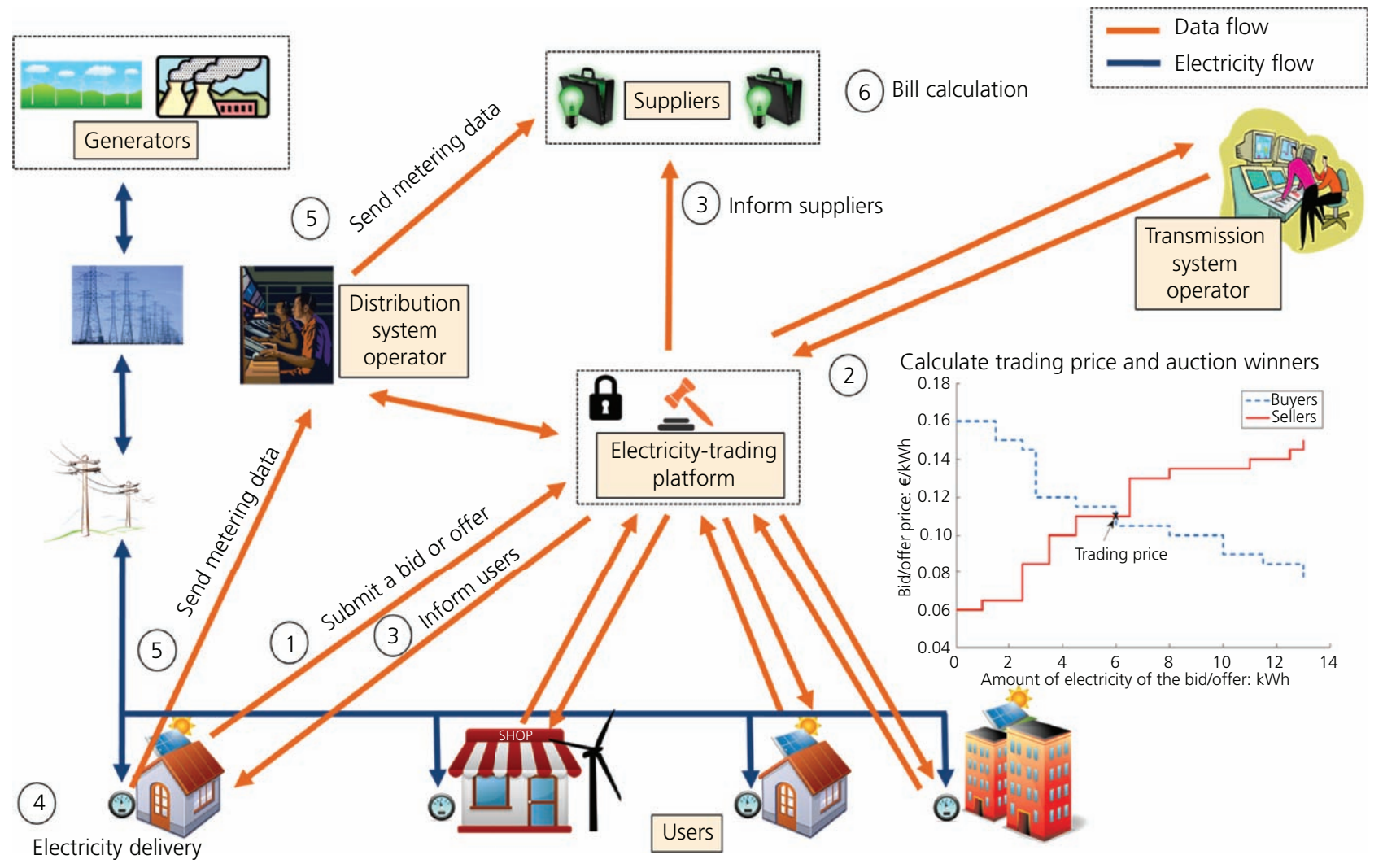

Figure 4. Peer-to-peer energy-trading scheme (Esat, 2019)

peer energy trading and on the role of blockchain in such applications, because they allow decentralising the application management as well.

\section{Blockchain in the context of peer-to-peer energy trading}

Centralised control schemes often rely on central actors, central data processing and central data storage. Due to the decentralisation of the power infrastructure, blockchain technology is considered an alternative in many energy applications (Andoni et al., 2019). Blockchains were introduced by Nakamoto (2008) together with Bitcoin, the first cryptocurrency. The blockchain is a data structure distributed among the participants in the network, called nodes. The data structure is a ledger that may contain digital transactions, data records and executables. The different steps of the process are as follows (Andoni et al., 2019).

- The nodes interact with the blockchain in a secure way by way of a public/private key pair. The use of this asymmetric encryption brings authentication and integrity into the network. Every signed transaction is broadcast to the one-hop peers of a node - that is, nodes one link further in the network.

- The neighbouring nodes verify the validity of these incoming transactions before retransmitting them any further. Eventually, this transaction is spread across the entire network.
- Transactions are then aggregated into blocks, which are timestamped and cryptographically linked to previous blocks, forming a chain of blocks, the blockchain. These blocks are broadcast back to the network by the nodes that formed them, the same way that the transactions are broadcast.

- The last step is for the network to verify that the block contains valid transactions and that the block is constructed correctly. If that is the case, the block is added to the chain, applying the transactions that it contains and updating the state of the network, providing a single source of truth available to all nodes in the network.

The steps described above are the basics of a blockchain network. There are many different implementations possible, with different performance and complexity trade-offs. The chosen consensus mechanism determines which nodes are allowed to propose blocks and how an agreement on which block to add to the chain is achieved between the nodes. This and other factors such as private against public networks lead to a taxonomy as introduced by Yeow et al. (2018).

Such distributed ledger principle looks promising as a secure, decentralised database for peer-to-peer energy transaction management. However, this is only a part of the solution. Once the data are stored and readily available, operations need to be applied to 
Smart Infrastructure and Construction

Volume 173 Issue 2
Digitalised, decentralised power

infrastructures challenge blockchains

Deconinck and Vankrunkelsven satisfy the needs of the different actors in the peer-to-peer market. Matching offers and demands to produce nominations, billing and charging grid tariffs are some examples. This is where smart contracts come into play, a concept introduced by Szabo (1997), where it was defined as 'a computerized transaction protocol that executes the terms of a contract'.

Contractual clauses are translated into code and are made selfenforcing. This can be embedded into property, which can be hardware or software, to eliminate the need for trusted intermediaries between transacting parties (Christidis and Devetsikiotis, 2016). An example in the peer-to-peer energytrading context would be a contract embedded in smart meters to perform electricity trading between peers.

A smart contract can be used in a blockchain context; in this case, the smart contract resides on the blockchain with a unique address or account. The contract is triggered by addressing a transaction to it. The content of the contract can then be executed by the nodes in the network based on the data from the transaction addressed to it. Combining smart contracts with blockchain technology opens up interesting possibilities for renewable energy trading (Mihaylov et al., 2018).

\section{Evaluation of a blockchain approach for peer-to-peer energy trading}

The dynamics of peer-to-peer energy trading have consequences for all market actors involved, from the system operator to the grid users. To make the discussion on market actors concrete, consider the existing market participants in Flanders (Belgium) as defined in Utility Market Implementation Guide (UMIG) version 6.5, Market Model UMIG 6.5.1.26 (Atrias, 2019), and shown in Figure 5. When introducing peer-to-peer energy trading, a possible implementation of the actors and their activities is described in Figure 6 (Vankrunkelsven, 2019).

For a more detailed analysis, the peer-to-peer energy trading is split up into five phases, as indicated in Figure 7 (Vankrunkelsven, 2019). In the market phase, bids and offers are continuously collected, resulting in one smart contract for each bid and offer. After the market clearing (e.g. every $15 \mathrm{~min}$ ), in the nomination phase, a smart contract is created for a balancing responsible party (e.g. a supplier), one for each customer that is going to sell or buy energy. In the delivery phase, one measurement for electricity injection and one for off-take is created per customer, such that the pre-agreed conditions of the smart contracts can be automatically checked during the allocation phase. Afterwards (e.g. daily or monthly), the remuneration phase translates the physical transactions into financial ones. This leads to a certain amount of blockchain actions as in Table 1. Assuming that all 3.4 million smart meters in Flanders would contribute in a single peer-to-peer energy-trading application allows estimating the performance and scalability in the following analysis.

The analysis consists of three main topics: scalability, costs, and privacy and security. For every topic, the specific blockchain implementation is discussed, in order to converge towards a specific blockchain architecture, for which its performance can be assessed.

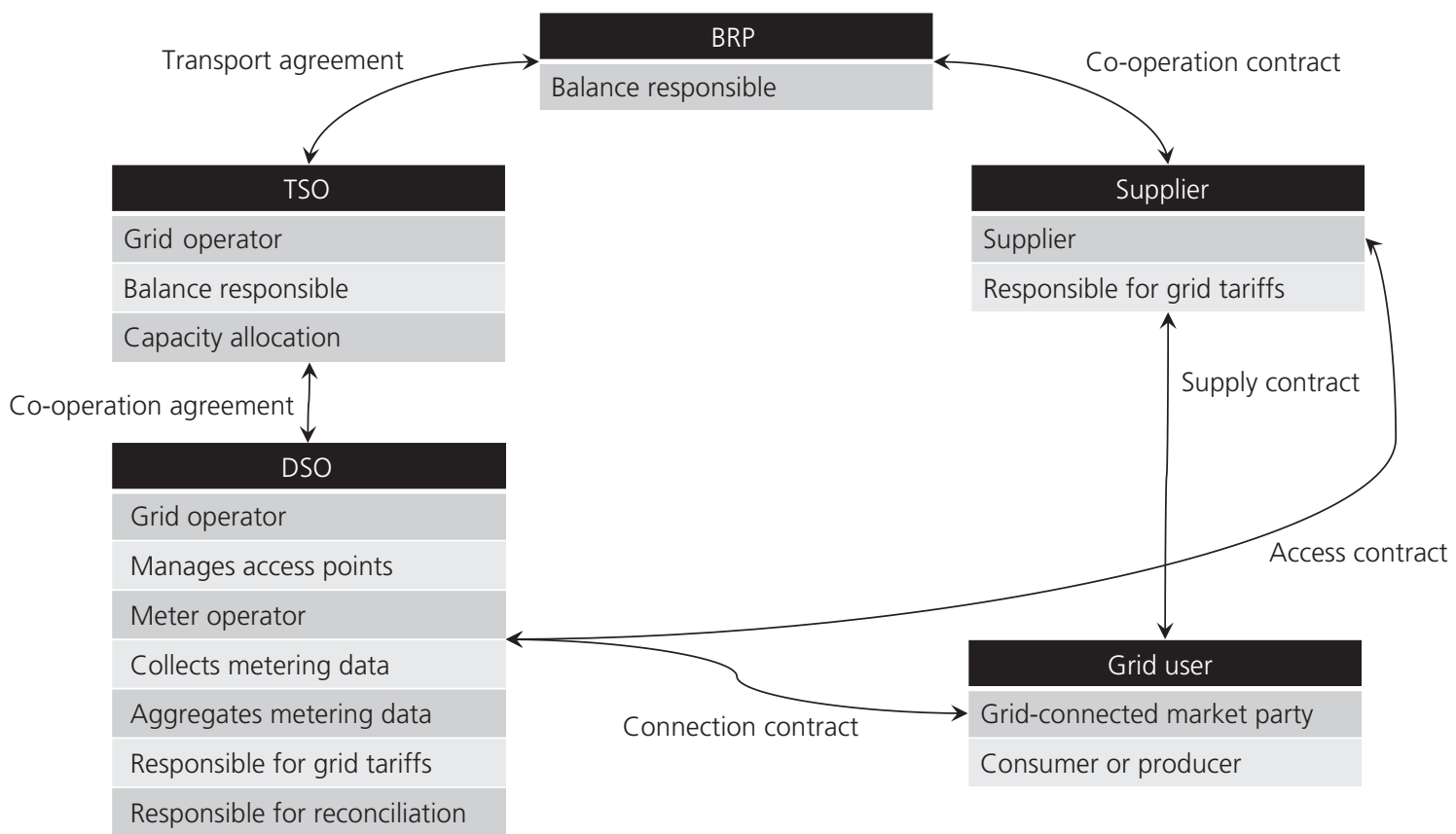

Figure 5. Market actors with their roles and mutual relations according to the UMIG by Atrias (2019). BRP, balance responsible party; TSO, transmission system operator 


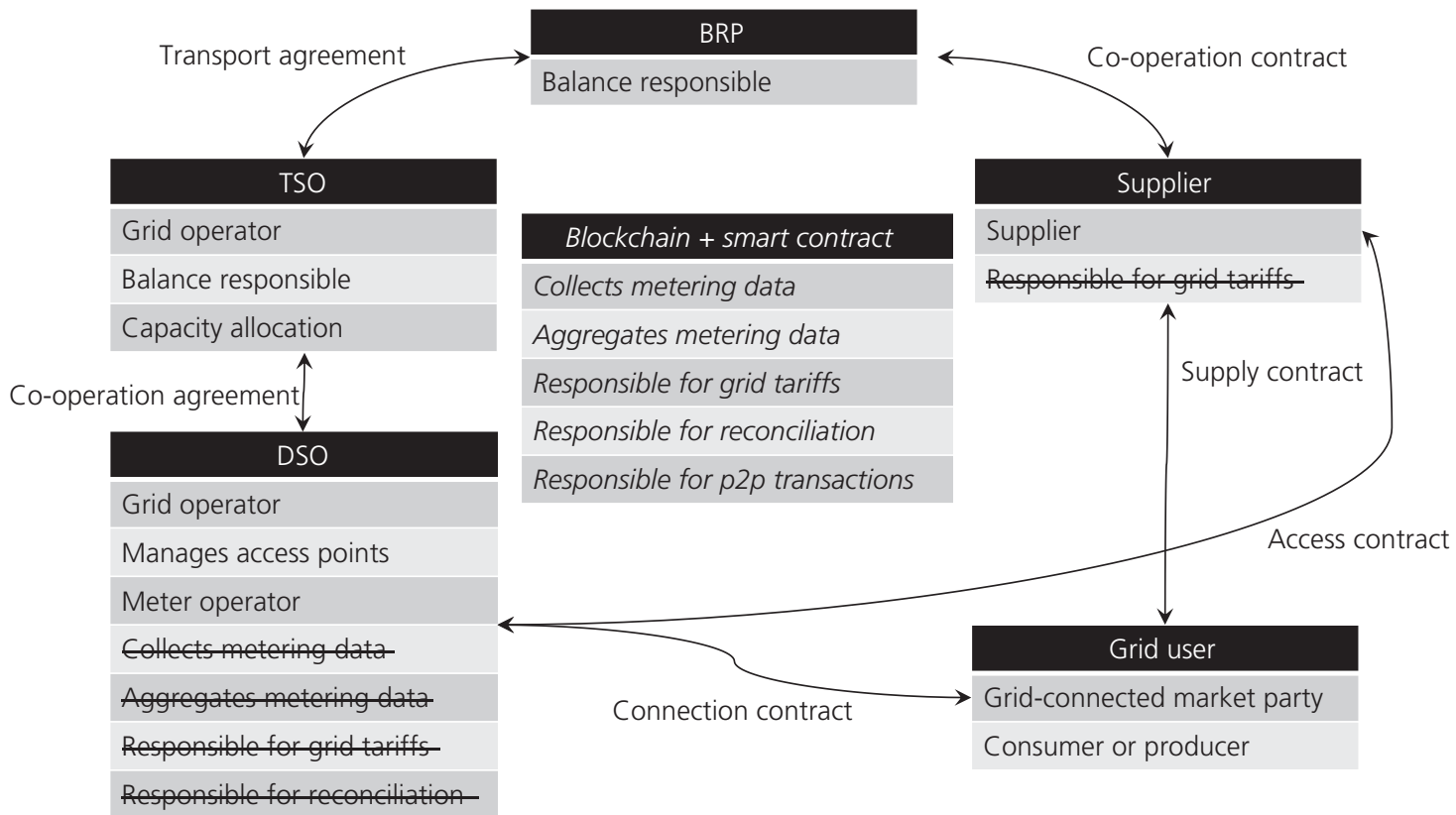

Figure 6. Market actors with their roles including blockchain and smart contracts, adapted from Figure 5. BRP, balance responsible party; p2p, peer-to-peer; TSO, transmission system operator (Vankrunkelsven, 2019)

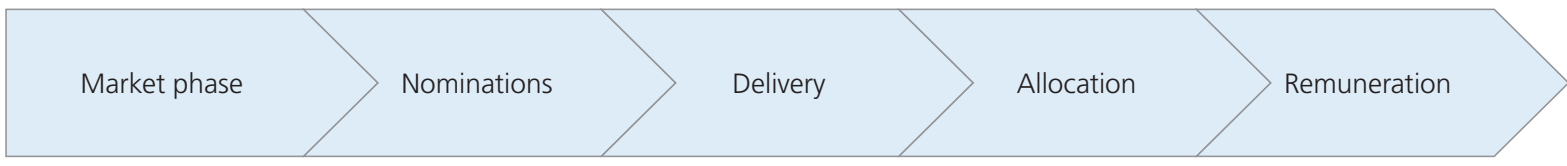

Figure 7. Schematic representation of the different phases of peer-to-peer energy trading (Vankrunkelsven, 2019)

Table 1. Overview of blockchain actions for different phases (Vankrunkelsven, 2019)

\begin{tabular}{lll} 
Time interval & \multicolumn{1}{c}{ Phase } & \multicolumn{1}{c}{ Action } \\
\hline Every $15 \mathrm{~min}$ & Market phase & One smart contract (SC) execution per bid or offer and possible price change of these \\
& Nominations & One SC execution per smart meter \\
& Delivery & One measurement per smart meter \\
Daily, monthly and so on & Allocation & One SC execution per smart meter \\
& Remuneration & One SC execution per smart meter, yielding multiple transactions
\end{tabular}

\subsection{Scalability}

According to the taxonomy of Yeow et al. (2018), a private, account-based blockchain model using smart contracts is chosen for the peer-to-peer energy-trading application. To work further towards a more detailed architecture, a first consideration is the trade-off between light nodes and full nodes (that do all blockchain calculations). A full node on the blockchain participates in all steps mentioned in the beginning of Section 3, while a light node participates in only some of these steps (e.g. skipping the validation step). These full nodes should be trusted by the light nodes and obtain permission to do the blockchain management on their behalf. As a smart meter can be considered a resource-constrained device, it can only act as a light node.
Light nodes do not contribute to the maintenance of the blockchain network but rely on the resources of full nodes for this. Hence, full nodes are still necessary to maintain the blockchain network. It would be a logical decision for the DSO to operate the full nodes. As such, the reward for operating a full node can easily be integrated in the grid tariff for the DSO. Otherwise, an extra value flow is needed. Additionally, as a full node operator, the DSO can set rules for the blockchain network, ensuring that only approved smart meters join the blockchain network as nodes, to set the correct distribution tariff (possibly differentiated), to make sure that energy transactions do not violate any grid constraints and so on. Such a permissioned blockchain where full nodes are operated by a few or a single 
trusted organisation(s) is called a consortium blockchain. It is often combined with a byzantine fault-tolerant (BFT) consensus mechanism that is able to deal with a more complex type of faults in a distributed system. Consensus mechanisms allow a majority of honest nodes to identify and overrule a minority of dishonest nodes in the network (Lamport et al., 2008).

To consider the performance, Table 1 shows that each step puts a certain burden on the system regarding transactions, smart contract executions and storage. The first four phases need to happen every $15 \mathrm{~min}$; therefore, $15 \mathrm{~min}$ can be seen as the maximum processing time. The remuneration step can be aggregated over a longer period of time without compromising the real-time benefits of peer-to-peer energy trading. The market phase, nominations, allocation and remuneration all require an interaction with a smart contract. The delivery step only needs to $\log$ data to the database.

To obtain a rough estimate of the amount of transactions per second, assume that the first four phases generate about five transactions per smart meter, for every smart contract at least one and some extra for price changes in the market phase. For 3.4 million meters and in $15 \mathrm{~min}$ intervals, this would result in about 19000 transactions per second (tps). Compared with the cryptocurrency Bitcoin (about 7 tps), Ethereum (another cryptocurrency; about 20 tps) or the blockchain implementation of the Energy Web Foundation (EWF, 2019) (which has been designed particularly for smart energy applications; about 600 tps), this amount is at the high end of the performance spectrum. Hence, it is worthwhile to look further into techniques to improve scalability or to investigate how the performance requirement can be lowered by splitting the energy-trading application into smaller ones, based on the hierarchical voltage structure of the electricity grid, with microgrids or local energy communities as subsystems.

Indeed, in the peer-to-peer context, local energy communities often have a limited scope where trading with neighbours or nearby family and friends on the same electrical feeder is favoured. This tendency is further amplified by local energy co-operatives, projects where communities jointly invest in renewable energy in the neighbourhood. By implementing, for example, side chains on such a subsystem level, the performance of the blockchain is matched. Distribution substations of the DSO at the edge of a low-voltage feeder can harbour full nodes maintaining a side chain managing this feeder as a subsystem. The side chain connects to the main chain covering microgrids all over Flanders. Other solutions might be used to unburden the blockchain even further, setting up transaction channels between neighbours often trading with each other (ConsenSys Media, 2018).

Regarding pure processing power, looking mainly at scalability, the amount of transactions per second, this work argues that there is no major benefit of using blockchain technology. In the paper by Andoni et al. (2019), it is stated that at this stage, relational databases, as currently used in centralised systems, have a decisive advantage when it comes to performance. However, a blockchain has an advantage when it comes to providing a robust, fault-tolerant way to store critical data and manage smart contracts.

\subsection{Cost and power efficiency of the blockchain implementation}

Many blockchain implementations are power-hungry, particularly due to the proof of work that has to be done by every full node on the blockchain. By combining full nodes with light nodes, which need less electrical energy per transaction, power consumption can be reduced.

Following the analysis further, the decision for a permissioned system with BFT consensus seems to be an efficient choice as well if compared with other consensus mechanisms. According to the EWF (2019), a consortium blockchain requires about $0.1 \mathrm{kWh}$ per transaction, which is, however, still about one order of magnitude greater than a centralised transaction.

From a cost perspective, assessment is difficult (Vankrunkelsven, 2019): as the blockchain is a distributed database, there are no central costs to operate and maintain this, but the database has to be built in the different full nodes, which all have to bear operation and maintenance costs.

Thus, from a power and cost perspective, there are few advantages associated with blockchain technology.

\subsection{Trust, privacy and security}

The advantages of the blockchain can rather be found in the trust, privacy and security aspects.

\subsubsection{Trust}

Blockchains and smart contracts allow users to trade without having to trust neither each other nor a third party. In this regard, the proofof-work concept fully empowers the full nodes, reaching complete decentralisation. Unfortunately, the poor computational performance of such proof of work, combined with the more than linearly increasing complexity of the consensus mechanism (in terms of numbers of nodes), forces some concessions in terms of decentralisation. Choosing the combination of full nodes and light nodes is a trade-off made in favour of scalability and at the cost of trust. However, by using the so-called Merkle tree root verification, light nodes are still able to authenticate full nodes correctly (Curran, 2020). If the DSO can be considered a trusted party, a permissioned, consortium type of blockchain with the DSO running the full nodes makes sense. It is not as if the DSO is now operating the market (this is done by smart contracts); the DSO is merely facilitating the platform and controlling who has access to it. Hence, this is still more trustable than a fully centralised approach.

\subsubsection{Privacy}

As every full node has a copy of the blockchain, all transactions can be followed transparently. On the one hand, this transparency is 
one of the strong points of blockchain; on the other hand, this presents challenges regarding privacy. Although blockchain nodes have an address that does not immediately reveal a user's identity (pseudonyms), all transactions with this address are visible on the blockchain. Studying the transactions of an address could hence link it to a real user. In a proof-of-work blockchain, this can be remedied to some extent by using different wallets to trade from. In the architecture chosen here for peer-to-peer energy trading, this is not possible as a smart meter has only one registered address.

Another challenge regarding privacy is compliance with the EU's GDPR (EC, 2016). On the one hand, users should be identifiable to account for their liabilities, but at the same time sensitive information needs to remain confidential. To give an idea of the challenge that a blockchain is facing, consider the following articles of legislation: article 16, right to rectification; article 17 , right to erasure; and article 18, right to restriction of processing. The fact that a blockchain is, in principle, immutable makes it very difficult to comply with articles 16 and 17. Further, knowing that all participating nodes have access to the data, everybody is able to do whatever they want with the data, violating article 18. A permissioned blockchain where access is restricted to authorised parties only is in this regard better than a permissionless one.

\subsubsection{Dependability and security}

Regarding dependability, a first line of defence is the redundancy provided by the blockchain. The distributed nature has no single point of failure; hence, it is more reliable than a centralised approach. Regarding security, a blockchain protects well against malicious intents, due to the immutable characteristics of the blockchain - that is, data cannot be tampered with retroactively. Therefore, new blocks need to be correct, which the consensus mechanism takes care of. In the BFT consensus, integrity is guaranteed as long as less than one-third of the nodes, full nodes in the peer-to-peer energy-trading case, are faulty or behave maliciously (Andoni et al., 2019). More centralised approaches reduce the amount of nodes participating in the mechanisms and thus reduce the absolute amount of nodes that are allowed to fail or behave dishonestly before compromising integrity.

Finally, asymmetric encryption ensures authorised access of registered meters only, as long as private keys are not compromised.

Hence, because of the distributed nature and cryptographic linking of the blocks, blockchain technology is considered more resistant to tampering than centralised systems. Additionally, the accountbased smart contract blockchain model provides a fully transparent ecosystem where users, their transactions and their data are verified in a secure yet accessible way. However, the privacy issues are non-negligible.

\section{Conclusion}

In a changing energy system where digitalisation and decentralisation are key drivers, peer-to-peer energy trading might be considered a fully automated and decentralised application. The first part of this paper positioned peer-to-peer energy trading within challenges related to the digitalisation and decentralisation of the energy system.

Within information technologies, many technological developments support this decentralisation; blockchain technology might be one piece of the puzzle. Peer-to-peer energy trading is considered one of many potential use cases where this technology can be applied.

The blockchain offers a robust digital platform, maintained in a distributed manner, suitable to organise a secure marketplace for electricity trading. With smart contracts, processes that are more complex can be automated in a fixed and unambiguous procedure. Electricity trade and flows are monitored, and payments are automatically processed in a secure and transparent way. This creates a marketplace that is also accessible to smaller market players, such as households and prosumers.

Moreover, the decentralised nature of the blockchain fits well with the renewable distributed energy resources that drive the peer-topeer market. However, blockchain technology is evolving very quickly. Many experimental projects and research are ongoing. Besides the financial sector (with cryptocurrencies), the energy sector has taken a big interest in the technology (EWF, 2019).

The second part of this paper investigated whether it is useful to apply blockchain technology in a peer-to-peer energy-trading context and what the benefits and drawbacks would be compared with those of current, centralised technologies. With numerous companies experimenting, the technology has passed its proof-ofconcept phase. However, large-scale projects are still lacking. It is now up to projects such as the Energy Web Chain of the EWF (2019), or similar ones, to make it happen on a commercial scale. Currently, full decentralisation with the desired amount of scalability and security seems hard to realise.

- This work showed that in order to meet the desired performance, more centralised types of the decentralised blockchain technology are required, such as a consortium blockchain where the third parties (e.g. the DSO) are still present and act as trusted operators of the platform. At the moment, the radical shift from centralised to decentralised seems to be stuck somewhere in between.

- The analysis regarding scalability shows that it is necessary to limit the amount of nodes maintaining the blockchain network, in order to achieve transaction speeds more practical than that of the currently established cryptocurrency blockchains. This can be realised by two main design choices.

- First, computing power can be centralised to some extent by choosing a system where full nodes do the heavy work and light nodes still allow resource-constrained nodes to participate.

- A second design choice is to limit the amount of parties operating these full nodes to a few authorised and trusted parties. This leads to the so-called consortium blockchain. 
- However, an estimation of the amount of transactions required for peer-to-peer energy trading in a region such as Flanders shows that the blockchain architecture is still underperforming. Blockchain approaches such as side chains and off-chain techniques could improve scalability, but they are still in the research stage.

v For the costs, there are too many cost elements that are hard to quantify or that are too sensitive to assumptions in order to compare the blockchain implementation with a centralised one.

- The final part of the analysis covered trust, privacy and security.

- The design choices for the scalability result in a system where still trust has to be put into third parties, such as DSOs. This cancels to some extent the complete trustlessness that other blockchains achieve, but it makes sense from a grid operation point of view.

- Regarding privacy, the transparent and immutable characteristics of the blockchain pose some problems when dealing with personal data according to the European GDPR. The choice for a consortium blockchain already helps control who has access. Further, zeroknowledge proofs and other encryption techniques are being developed to comply with privacy regulations.

- Lastly, the system security that the blockchain offers seems to be the major advantage. The way that the integrity and trustworthiness of the data is maintained automatically, through encryption and distributed consensus for large amounts of devices, could become very important in an increasingly interconnected energy infrastructure.

- Where possible, a comparison with a centralised system was made. The literature often describes centralised approaches introducing more hierarchical systems where the data, the market or another application is handled on multiple levels. Introducing agents or data-management devices at multiple levels allows for more efficient processing. This also results in a form of decentralisation.

It seems that centralised systems and completely distributed blockchain systems are evolving towards each other for use in the energy sector. Future research and developments will point out where the two will meet. Performance, scalability, cost, privacy and security are crucial factors in this comparison.

\section{Acknowledgements}

This research has partially been supported by the Fund for Scientific Research - Flanders by way of project FWO-SBOS007619N-SNIPPET (Secure aNd prIvacy-friendly Peer-to-Peer Electricity Trading).

This work is an extended version of the abstract presented at International Symposium for Next Generation Infrastructure 2019
(Deconinck et al., 2019) and is partially based on the MSc thesis of the second author (Vankrunkelsven, 2019).

\section{REFERENCES}

Abdella J and Shuaib K (2018) Peer to peer distributed energy trading in smart grids: a survey. Energies 11(6): article 1560, https://doi.org/10. 3390/en11061560.

Almasalma H, Claeys S and Deconinck G (2019) Peer-to-peer-based integrated grid voltage support function for smart photovoltaic inverters. Applied Energy 239: 1037-1048, https://doi.org/10.1016/J. APENERGY.2019.01.249.

Andoni M, Robu V, Flynn D et al. (2019) Blockchain technology in the energy sector: a systematic review of challenges and opportunities. Renewable and Sustainable Energy Reviews 100: 143-174, https://doi. org/10.1016/j.rser.2018.10.014

Argentina.gob.ar (2019) PERMER: Proyecto de Energías Renovables en Mercados Rurales. Argentina.gob.ar, Buenos Aires, Argentina (in Spanish). See https://www.argentina.gob.ar/energia/permer (accessed $13 / 12 / 2019)$

Atrias (2019) ATRIAS Market Model UMIG 6.5.1.26. Atrias, Brussels, Belgium. See http://www.atrias.be/UK/Pages/Publications_UMIG65. aspx (accessed 13/12/2019).

CCC (Chaos Computer Club e.V.) (2020) See https://www.ccc.de/en/home (accessed 05/05/2020).

Cherepanov A and Lipovsky R (2017) Industroyer: biggest threat to industrial control systems since Stuxnet. WeLiveSecurity, June. See https://www.welivesecurity.com/2017/06/12/industroyer-biggest-threatindustrial-control-systems-since-stuxnet/ (accessed 21/11/2020).

Christidis K and Devetsikiotis M (2016) Blockchains and smart contracts for the Internet of Things. IEEE Access 4: 2292-2303, https://doi.org/ 10.1109/ACCESS.2016.2566339.

ConsenSys Media (2018) The state of scaling Ethereum. ConsenSys Media, 25 April. See https://media.consensys.net/the-state-of-scalingethereum-b4d095dbafae (accessed 06/05/2020).

Curran B (2020) What is a Merkle tree? Beginner's guide to this blockchain component. Blockonomi, 18 April. See https://blockonomi. com/merkle-tree/ (accessed 06/05/2020).

Deconinck G and Thoelen K (2019) Lessons from 10 years of demand response research: smart energy for customers? IEEE Systems, Man, and Cybernetics Magazine 5(3): 21-30, https://doi.org/10.1109/ MSMC.2019.2920160.

Deconinck G and Vanthournout K (2009) Agora: a semantic overlay network. International Journal of Critical Infrastructures 5(1/2): 175-195, https://doi.org/10.1504/IJCIS.2009.022855.

Deconinck G, Vankrunkelsven F and Patyn C (2019) On the added value of smart contracts in peer-to-peer electricity trading. Proceedings of the 2019 International Symposium on Next Generation Infrastructure, Buenos Aires, Argentina.

EC (European Community) (2016) Regulation (EU) 2016/679 of the European Parliament and of the Council of 27 April 2016 on the protection of natural persons with regard to the processing of personal data and on the free movement of such data, and repealing Directive 95/46/EC (General Data Protection Regulation). Official Journal of the European Union L119/1. See https://eur-lex.europa.eu/eli/reg/2016/ 679/oj (accessed 06/05/2020).

EC (2018) Directive (EU) 2018/2001 of the European Parliament and of the Council of 11 December 2018 on the promotion of the use of energy from renewable sources. Official Journal of the European Union L328/82.

EC (2019) Directive (EU) 2019/944 of the European Parliament and of the Council of 5 June 2019 on common rules for the internal market for electricity and amending Directive 2012/27/EU. Official Journal of the European Union L158/125. See http://data.europa.eu/eli/dir/2019/944/ oj (accessed 20/11/2020). 
electricityMap (2020) See https://www.electricitymap.org (accessed 11/09/2019).

Esat (Department of Electrical Engineering, KU Leuven) (2019) SNIPPET: Secure and Privacy-friendly Peer-to-peer Electricity Trading. Esat, Leuven, Belgium. See https://www.esat.kuleuven.be/cosic/project/ snippet/ (accessed 10/07/2019).

EWF (Energy Web Foundation) (2019) The Energy Web Chain: Accelerating the Energy Transition with an Open-source, Decentralized Blockchain Platform. EWF, Berlin, Germany. See https://www.energyweb.org/reports/ the-energy-web-chain/ (accessed 21/11/2020).

Flemish Government (2013) Decreet tot wijziging van het decreet van 20 december 1996 tot regeling van het recht op minimumlevering van elektriciteit, gas en water en tot wijziging van het decreet van $24 \mathrm{mei}$ 2002 betreffende water bestemd voor menselijke aanwending, wat de minimumlevering van water betreft en de bescherming van abonnees tegen afsluiting van de drinkwatertoevoer. Flemish Government, Brussels, Belgium (in Dutch). See https://codex.vlaanderen.be/Zoeken/ Document.aspx?DID=1023209 (accessed 21/11/2020).

Haapola J, Ali S, Kalalas C et al. (2018) Peer-to-peer energy trading and grid control communications solutions' feasibility assessment based on key performance indicators. Proceedings of the 2018 IEEE 87th Vehicular Technology Conference (VTC Spring), Porto, Portugal, pp. 1-5.

Khan R, Maynard P, McLaughlin K, Laverty D and Sezer S (2016) Threat analysis of BlackEnergy malware for synchrophasor based real-time control and monitoring in smart grid. In 4th International Symposium for ICS \& SCADA Cyber Security Research 2016 (Janicke H, Jones K and Brandstetter T (eds)). BCS Learning \& Development, Swindon, UK, pp. 53-63.

Labeeuw W and Deconinck G (2011) Non-intrusive detection of high power appliances in metered data and privacy issues. Proceedings of the 25th Conference on Passive and Low Energy Architecture of the Energy Efficiency in Domestic Appliances and Lighting (EEDAL 2011), Copenhagen, Denmark, pp. 1-7.

Lamport L, Shostak R and Pease M (2008) The Byzantine generals problem. Dr. Dobb's Journal 33(4): 30-36, https://doi.org/10.1145/ 3335772.3335936

Liu CC, McArthur S and Lee SJ (eds) (2016) Smart Grid Handbook. Wiley, Chichester, UK.

Mee Panyar (2019) See https://www.meepanyar.com/ (accessed 13/12/2019).

Mihaylov M, Razo-Zapata I and Nowé A (2018) NRGcoin - a blockchainbased reward mechanism for both production and consumption of renewable energy. In Transforming Climate Finance and Green Investment with Blockchains (Marke A (ed.)). Academic Press, London, UK, pp. 111-131.
Montakhabi M, Zobiri F, van der Graaf S et al. (2020) New roles in peerto-peer electricity markets: value network analysis. In Proceedings of the 2020 6th IEEE International Energy Conference (EnergyCon), Gammarth, Tunisia, pp. 389-394.

Nakamoto S (2008) Bitcoin: a Peer-to-peer Electronic Cash System, bitcoin.org. See https://www.bitcoin.org/bitcoin.pdf (accessed 05/05/ 2020).

REScoop.eu (2019a) See https://www.rescoop.eu/ (accessed 12/07/2019).

REScoop.eu (2019b) Q \& A: What are 'citizen' and 'renewable' energy communities? REScoop.eu, 3 July. See https://www.rescoop.eu/blog/ what-are-citizen-and-renewable-energy-communities (accessed 04/07/2019).

Roberts J and Gauthier C (2019) Energy Communities in the Draft National Energy and Climate Plans: Encouraging but Room for Improvements, European University Viadrina, REScoop.eu and Friends of the Earth Europe, Brussels, Belgium. See https://www.rescoop.eu/ blog/necps (accessed 06/05/2020).

SCADAhacker (2020) https://www.scadahacker.com/ (accessed $18 / 09 / 2020)$

Scott J (2009) Smart grids - the European technology platform for electricity networks of the future. In E-Energy (Picot A and Neumann K (eds)). Springer, Berlin, Germany, pp. 11-23.

Szabo N (1997) Formalizing and securing relationships on public networks. First Monday 2(9), https://doi.org/10.5210/fm.v2i9.548.

UNDP (UN Development Programme) (2015) Sustainable Development Goal 7: Affordable and Clean Energy. UNDP, New York, NY, USA. See https://www.undp.org/content/undp/en/home/sustainabledevelopment-goals/goal-7-affordable-and-clean-energy.html (accessed 14/05/2020).

UsersTCP (Users Technology Cooperation Programme) (2020) Global Observatory on Peer-to-peer Community Self-consumption and Transactive Energy Models. UsersTCP. Paris, France. See https:// userstcp.org/annex/peer-to-peer-energy-trading/ (accessed 05/05/2020).

Vankrunkelsven F (2019) Evaluation of the Added Value of Smart Contracts on Top of Blockchain for a Distributed Peer-to-peer Electricity Market - a Comparison with the Existing Centralised Approach in a Belgian Context. MSc thesis, KU Leuven, Leuven, Belgium.

Vanthournout K, De Brabandere K, Heasen E et al. (2005) Agora: distributed tertiary control of distributed resources. Proceedings of the 15th Power Systems Computation Conference (PSCC 2005), Liège, Belgium, paper no. 5.

Yeow K, Gani A, Ahmad RW, Rodrigues JJPC and Ko K (2018) Decentralized consensus for edge-centric Internet of Things: a review, taxonomy, and research issues. IEEE Access 6: 1513-1524, https://doi. org/10.1109/ACCESS.2017.2779263.

\section{How can you contribute?}

To discuss this paper, please email up to 500 words to the editor at journals@ice.org.uk. Your contribution will be forwarded to the author(s) for a reply and, if considered appropriate by the editorial board, it will be published as discussion in a future issue of the journal.

Proceedings journals rely entirely on contributions from the civil engineering profession (and allied disciplines). Information about how to submit your paper online is available at www.icevirtuallibrary.com/page/authors, where you will also find detailed author guidelines. 